\title{
Multicenter phase III trial of S-1 and cisplatin versus S-1 and oxaliplatin combination chemotherapy for first-line treatment of advanced gastric cancer (SOPP trial)
}

\author{
Keun-Wook Lee ${ }^{1} \cdot$ Ik-Joo Chung $^{2} \cdot$ Min-Hee Ryu ${ }^{3}$. Young lee Park ${ }^{4} \cdot$ Byung-Ho Nam $^{5,12} \cdot$ Ho-Suk Oh $^{6}$. \\ Kyung Hee Lee ${ }^{7}$. Hye Sook Han ${ }^{8}$. Bong-Gun Seo ${ }^{9}$. Jae-Cheol Jo ${ }^{10}$. Hyo Rak Lee ${ }^{11}$. Jin Won Kim ${ }^{1}$. Sook Ryun Park ${ }^{3}$. \\ Sang Hee $\mathrm{Cho}^{2} \cdot$ Yoon-Koo $\mathrm{Kang}^{3}$ on behalf of the SOPP study investigators
}

Received: 2 March 2020 / Accepted: 13 June 2020 / Published online: 28 June 2020

(c) The International Gastric Cancer Association and The Japanese Gastric Cancer Association 2020

\begin{abstract}
Background In East Asia, S-1 plus cisplatin (SP) is one of the standard first-line chemotherapy regimens for metastatic or recurrent gastric cancer (MRGC). Oxaliplatin is generally less toxic and more convenient to administer than cisplatin.

Patients and methods This was a multicenter, phase III study assessing whether S-1/oxaliplatin (SOX) was non-inferior/ superior to SP in terms of progression-free survival (PFS). Patients with MRGC were randomized 1:1 to receive either SOX (S-1 $80 \mathrm{mg} / \mathrm{m}^{2} /$ day on days $1-14$; oxaliplatin $130 \mathrm{mg} / \mathrm{m}^{2}$ on day 1 ; every 3 weeks) or SP (S-1 $80 \mathrm{mg} / \mathrm{m}^{2} /$ day on days $1-14$; cisplatin $60 \mathrm{mg} / \mathrm{m}^{2}$ on day 1 ; every 3 weeks [SP3]).

Results Between October 2012 and October 2014, 338 patients were randomized. The median age was 56 years, and 51\% of patients had measurable lesions. SOX was significantly non-inferior but not superior to SP3 in terms of PFS [median 5.6 versus 5.7 months; hazard ratio (HR) 0.85 ; $95 \%$ confidence interval (CI) $0.67-1.07]$. In patients with measurable disease, objective response rates were similar between SOX and SP3 (58\% versus 60\%). Overall, the survival in both groups did not differ (median 12.9 versus 11.4 months; HR 0.86 ; 95\% CI 0.66-1.11). Treatment was well tolerated in both arms. Anemia, leucopenia, neutropenia, febrile neutropenia, and oral mucositis were more common with SP3. In contrast, thrombocytopenia, nausea, vomiting, and peripheral neuropathy were more common with SOX.

Conclusions SOX was non-inferior to SP3. The two regimens were well tolerated with different toxicity profiles. The SOX regimen can be recommended as a first-line treatment for MRGC.
\end{abstract}

Trial registration ClinicalTrials.gov: NCT01671449

Keywords Gastric cancer · S-1 · Oxaliplatin · Cisplatin · Phase III · Chemotherapy

Keun-Wook Lee and Ik-Joo Chung contributed equally.

Electronic supplementary material The online version of this article (https://doi.org/10.1007/s10120-020-01101-4) contains supplementary material, which is available to authorized users.

Min-Hee Ryu

miniryu@amc.seoul.kr

Young Iee Park

youngiee@ncc.re.kr

Extended author information available on the last page of the article

\section{Introduction}

Gastric cancer (GC) is the fifth most common malignancy and the third most common cause of cancer-related deaths worldwide [1]. In localized GC, surgery is the curative treatment modality; however, postoperative recurrence is common and observed in situations where attempting a curative treatment approach is difficult. In most cases of metastatic GC, the metastasis is usually widespread at the time of initial diagnosis. Hence, a curative treatment approach is almost impossible. Therefore, treatment for metastatic or recurrent GC (MRGC) remains a significant challenge.

Palliative chemotherapy is a cornerstone of treatment for patients with MRGC and is known to prolong overall survival (OS) and improve the quality of life (QOL) compared 
with best supportive care only [2]. The standard first-line chemotherapy for MRGC is based on fluoropyrimidine plus platinum combination, with trastuzumab added for HER2 + tumors [2, 3]. Among platinum agents, oxaliplatin has proven to be as effective as cisplatin when combined with 5-fluorouracil or capecitabine [4, 5]; as oxaliplatin causes less emesis and does not require large amounts of fluid infusion for renal protection compared with cisplatin, oxaliplatin has been replacing cisplatin in many cases with GC. Among fluoropyrimidine agents, both oral agents, capecitabine and S-1, are non-inferior to intravenous 5-fluorouracil and thus can replace 5-fluorouracil [5-7].

S-1 is an oral agent consisting of tegafur (5-fluorouracil prodrug), 5-chloro-2,4-dihydroxypyridine (an inhibitor of dihydropyrimidine dehydrogenase), and potassium oxonate (an inhibitor of the phosphorylation of 5-fluorouracil in the gastrointestinal tract). In a Japanese phase III study (JCOG9912), S-1 was proven to be non-inferior to 5-fluorouracil as monotherapy in MRGC [7]. Additionally, in another Japanese phase III study (SPIRITS), the combination of S-1 and cisplatin (SP) demonstrated a superior OS than S-1 monotherapy [8]. Based on these studies, SP has been widely used as one of the standard first-line therapies for patients with MRGC, especially in East Asian countries. However, in Korea, a 3-weekly SP regimen (SP3) is more commonly used than the Japanese 5-weekly SP (SP5) regimen [9]. Recently, the efficacy of S-1 plus oxaliplatin (SOX) regimens have been actively investigated. Several phase II studies have tested various doses and schedules of SOX regimens as first-line therapy in MRGC and have reported an objective response rate (ORR) of 30-59\%, progression-free survival (PFS) of 4.4-7.0 months, and OS of 7.8-16.5 months [10-15]. Therefore, a phase III SOPP study was performed to verify non-inferiority or superiority of SOX compared to SP3 in terms of PFS, using the 3-weekly schedule of both regimens, in Korean patients with MRGC.

\section{Methods}

\section{Study design}

This study was a multicenter, open-label, randomized, noninferiority phase III trial, conducted at 10 Korean institutions. Eligible subjects were randomly assigned to receive either SOX or SP3 in a 1: 1 ratio using permuted block randomization via interactive web response services. Three stratification factors were used as follows: (1) disease status (recurrence after curative gastrectomy versus initially unresectable or metastatic disease without gastrectomy versus initially curatively unresectable or metastatic disease with palliative gastrectomy; (2) presence of measurable disease; and (3) institution. The randomization results of the allocated study treatments were open to the investigators and patients.

This study was conducted in compliance with the Declaration of Helsinki and Good Clinical practice guidelines. The study was approved by the institutional review boards at each center. All patients gave their written informed consent and this trial was registered with ClinicalTrials.gov (NCT01671449).

\section{Patient eligibility criteria}

The inclusion criteria were as follows: patients with unresectable locally advanced, metastatic or recurrent adenocarcinoma of the stomach; age of $\geq 18$ years; Eastern Cooperative Oncology Group (ECOG) performance status (PS) $0-2$; the existence of at least one measurable or evaluable lesion (according to Response Evaluation Criteria In Solid Tumors (RECIST, version 1.1); adequate bone marrow, liver, and kidney function; life expectancy of $>3$ months; and no previous chemotherapy for MRGC. If a patient with resectable GC had received non-platinum-based neoadjuvant or adjuvant chemotherapy and the tumor recurred $>6$ months after the completion of neoadjuvant or adjuvant chemotherapy, the patient could be enrolled in this study. Patients with recent major surgery or radiotherapy within 4 weeks of randomization, central nervous system metastasis, or other serious comorbidities were excluded.

\section{Study treatment}

In SOX therapy, patients received intravenous oxaliplatin $\left(130 \mathrm{mg} / \mathrm{m}^{2}\right.$ over $2 \mathrm{~h}$; day 1$)$ followed by oral administration of S-1 (40 mg/m² ${ }^{2}$, twice daily; days 1-14). In SP3, cisplatin $\left(60 \mathrm{mg} / \mathrm{m}^{2}\right)$ was administered intravenously for $1 \mathrm{~h}$ on day 1 with adequate hydration, and $\mathrm{S}-1\left(40 \mathrm{mg} / \mathrm{m}^{2}\right.$; twice daily) was administered on days 1-14. In both arms, the calculation of S-1 dosage was based on body surface area (BSA) and the range of the $S-1$ dosage $\left(80 \mathrm{mg} / \mathrm{m}^{2} /\right.$ day) was $100-160 \mathrm{mg} /$ day (Supplementary Table 1). The study treatment was repeated every 3 weeks and continued until progressive disease (PD) according to the local investigator's assessment, unacceptable adverse effects (AEs), or withdrawal of consent.

In both arms, the dose of each anticancer drug was appropriately reduced based on the degree of AEs that developed during the previous cycle. If a severe hematologic $\mathrm{AE}$ developed, the administration of S-1 and cisplatin (or oxaliplatin) was delayed until recovery to absolute neutrophil count $>1.5 \times 10^{9} / \mathrm{L}$ and platelet count $>100 \times 10^{9} / \mathrm{L}$, and then $\mathrm{S}-1$ and cisplatin (or oxaliplatin) were administered at a one-dose-level reduction. If an investigator deemed that the AE was only caused by one drug (for example, nephrotoxicity or neuropathy due to cisplatin), no dose modifications for 
the other drug were required. Even if cisplatin or oxaliplatin was permanently discontinued due to AEs, S- 1 was allowed to continue until PD. However, the administration of only cisplatin or oxaliplatin without S-1 was not permitted under any circumstances. Guidelines for dose reduction according to the hematologic or non-hematologic AEs are detailed in Supplementary Table 1.

\section{Assessments}

The primary endpoint of the study was PFS, defined as the time from the date of randomization to the date of documented PD or death from any cause, whichever occurred first. According to the RECIST, tumor assessments using computed tomography $(\mathrm{CT})$ of the abdomen ( \pm chest) were performed every 2 cycles ( 6 weeks: window, \pm 7 days) from randomization until PD. Patients whose study treatment was terminated for reasons other than PD underwent a tumor assessment every 6 weeks before PD or subsequent secondline chemotherapy. All CT images for tumor responses and PFS were reviewed by the investigators. OS was defined as the time from randomization to death from any cause. AEs were classified according to the Common Terminology Criteria for Adverse Events (CTCAE; version 4.03). QOL was measured using the 3-level version of EuroQoL 5-dimensional instrument (EQ-5D-3L) at the baseline, every 2 cycles (at the time of tumor assessment), and at the end of study treatment. The EQ-5D-3L comprises a short descriptive system questionnaire on 5 distinct dimensions (mobility, selfcare, usual activities, pain/discomfort, and anxiety/depression) and a visual analog scale (EQ VAS); each dimension in the questionnaire has 3 levels (indicating no problem, some or moderate problem, and extreme problem) [16].

\section{Statistical considerations}

Efficacy and safety analyses were conducted in patients included in the full analysis set (FAS), which is a group that could meet the intention-to-treat principle. The FAS included all randomized patients except those who met the following criteria: subjects who violated inclusion or exclusion criteria, subjects without any information after randomization, or subjects who did not receive any study treatment after randomization. Among patients included in the FAS, those who had completed at least one cycle of treatment and had at least one tumor response evaluation were included in the per-protocol set (PPS).

The primary endpoint of this study was PFS and secondary endpoints were OS, ORR, QOL, and safety. The primary objective of this study was to demonstrate the non-inferiority of 3-weekly SOX in terms of PFS when compared with SP3. A non-inferiority margin of 1.4 , power $=80 \%$, one-sided $\alpha=0.025$, accrual period of 24 months, follow-up period of
12 months, expected median PFS in SP3 $=6$ months were expected. Based on these conditions, a total of 277 events were needed and a total of 304 subjects were required. Considering a $10 \%$ loss to follow-up, the enrollment of 338 subjects was finally planned.

Sequential tests for non-inferiority were planned with a non-inferiority margin of 1.4 and 1.25 . In the first step, a test for non-inferiority margin of 1.4 was conducted, and if the null hypothesis was rejected, then it was planned to conduct the second test to verify whether it would satisfy the non-inferiority margin of 1.25 . If non-inferiority was confirmed with the non-inferiority margin of 1.25 , then a test for superiority of SOX to SP3 was planned. The Kaplan-Meier method was used to estimate the PFS distribution, and the Cox proportional hazards regression model was used to calculate the hazard ratio (HR) and adjust the influence of prognostic and confounding parameters on PFS. Regarding sub-group analysis, univariable Cox regression model was used to estimate the HR in each group with respect to PFS and OS. Student's t-test was performed to compare means, and Chi-square test was used to compare percentages in cross tabulations. $P$-values $<0.05$ were considered statistically significant. Statistical analyses were conducted using SAS (version 9.3; SAS Institute, Inc., Cary, NC).

\section{Results}

\section{Patient characteristics}

From October 2012 to October 2014, 338 patients were randomized. One patient who was assigned to the SP3 group immediately withdrew consent and did not receive the study treatment; therefore, the FAS for efficacy and safety analyses included 337 patients (Fig. 1). Overall, the clinical characteristics were well balanced between SOX and SP3 (Table 1). Although the age of patients in the SOX group tended to be higher than that in the SP3 group, this was not statistically significant. Of the 337 patients in FAS, 304 were included in the PPS.

\section{Delivery of study treatments}

The analyses of treatment delivery and dose intensities (DIs) were conducted in PPS. In the SOX regimen, the median treatment cycles of S-1 and oxaliplatin were 6 (range 1-45) and 5 (1-30), respectively. In the SP3 regimen, median treatment cycles of S-1 and cisplatin were 5 (range 1-35) and 5 (1-31), respectively. In SOX, dose reductions of S-1 and oxaliplatin were conducted in $70 \%$ and $70 \%$ of patients, respectively. In SP3, dose reductions in S-1 and cisplatin were conducted in $72 \%$ and $71 \%$, respectively. In SOX, the relative DIs (RDIs) of S-1 and oxaliplatin were $80 \%( \pm 14)$ 


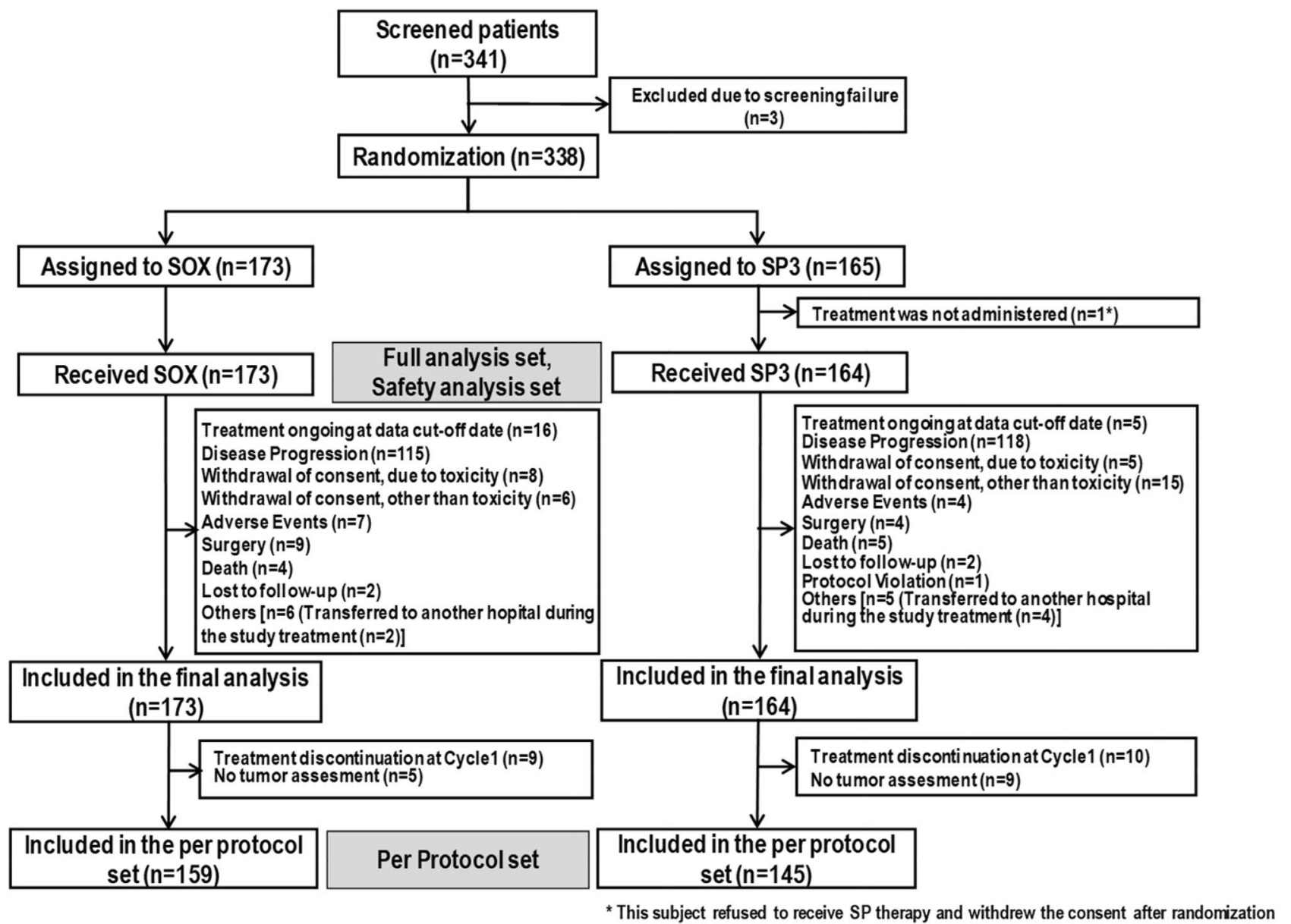

Fig. 1 CONSORT diagram (SOX, S-1 plus oxaliplatin; SP3, S-1 plus cisplatin)

(mean \pm standard deviation) and $84 \%( \pm 12)$, respectively. In SP3, the RDIs of S-1 and cisplatin were $77 \%( \pm 16)$ and $82 \%$ $( \pm 12)$, respectively (Supplementary Table 2).

Of the 173 patients in SOX (FAS), 100 patients (58\%) received second-line therapy after the discontinuation of SOX [taxane-based $(N=50)$, irinotecan-based $(N=36)$, and others $(N=14)]$. In SP3, $101(62 \%)$ of the 164 patients received second-line chemotherapy [taxane-based $(N=39)$, irinotecan-based $(N=38)$, and others $(N=24)]$.

\section{Efficacy}

The duration of the median follow-up was 15.6 months (range 0.1-33.3) in surviving patients as of the data cut-off date (Dec 23, 2015). The median PFS with the SOX and SP3 regimens were 5.6 months [95\% confidence interval (CI), 4.4-6.9] and 5.7 months [95\% CI 4.9-6.7], respectively (Fig. 2a). The unadjusted HR of SOX over SP3 was 0.85 (95\% CI 0.67-1.07). Since the upper margin of the HR was 1.07 , which was below the non-inferiority margin of 1.25 , the non-inferiority of SOX was confirmed with both non-inferiority margins of 1.4 and 1.25 . When the Cox regression model was adjusted for stratification factors (disease status at enrollment, presence of measurable disease, and institution), the adjusted HR was 0.90 (95\% CI $0.70-1.16)$. In the final step, a superiority test was conducted; however, the superiority of SOX over SP3 was not proven.

The median OS with SOX and SP3 treatments was 12.9 months (95\% CI 10.3-14.6) and 11.4 months (95\% CI 9.9-12.4) (Fig. 2b). The unadjusted and adjusted HRs of SOX over SP3 was 0.86 (95\% CI 0.66-1.11; $P=0.242$ ) and 0.84 (95\% CI $0.63-1.11 ; P=0.223$ ), respectively. Tumor response evaluation was performed in patients with measurable lesions $(N=173)$. ORRs with SOX and SP3 treatments were $58 \%$ and $60 \%(P=0.700)$, respectively. Disease control rates with SOX and SP3 regimens were $79 \%$ and $81 \%$ $(P=0.725)$, respectively (Supplementary Table 3$)$.

Subgroup analyses of PFS and OS are shown in Supplementary Fig. 1. Overall, between SOX and SP3, no difference in PFS or OS were observed, except subgroups of body mass index $(\mathrm{BMI}) \geq 25$ and age $\geq 60$ years. In patients 
Table 1 Patient characteristics

\begin{tabular}{|c|c|c|c|}
\hline & $\operatorname{SOX}(N=173)$ & $\mathrm{SP} 3(N=164)$ & $P$-value \\
\hline Gender & & & $0.204^{\mathrm{a}}$ \\
\hline Male & $123(71 \%)$ & $106(65 \%)$ & \\
\hline Female & $50(29 \%)$ & $58(35 \%)$ & \\
\hline Age, median (range) & $58(29-80)$ & $55(26-78)$ & $0.051^{\mathrm{b}}$ \\
\hline Body mass index, median (range) & $21.9(14.1-31.6)$ & $21.6(14.3-34.9)$ & $0.267^{\mathrm{b}}$ \\
\hline ECOG performance status & & & $0.372^{\mathrm{a}}$ \\
\hline 0 & $41(24 \%)$ & $29(18 \%)$ & \\
\hline 1 & $129(75 \%)$ & $131(80 \%)$ & \\
\hline 2 & $3(2 \%)$ & $4(2 \%)$ & \\
\hline Disease status at enrollment & & & $0.326^{\mathrm{a}}$ \\
\hline Recurrence after curative gastrectomy & $28(16 \%)$ & $27(16 \%)$ & \\
\hline Initially metastatic disease without gastrectomy & $128(74 \%)$ & $128(78 \%)$ & \\
\hline Initially metastatic disease with gastrectomy & $17(10 \%)$ & $9(5 \%)$ & \\
\hline Disease extent at enrollment & & & $0.907^{\mathrm{a}}$ \\
\hline Locally advanced and unresectable & $9(5 \%)$ & $9(5 \%)$ & \\
\hline Metastatic & $164(95 \%)$ & $155(95 \%)$ & \\
\hline Prior adjuvant chemotherapy & & & $0.727^{\mathrm{a}}$ \\
\hline Yes & $11(6 \%)$ & $12(7 \%)$ & \\
\hline No & $162(94 \%)$ & $152(93 \%)$ & \\
\hline Presence of measurable disease & & & $0.487^{\mathrm{a}}$ \\
\hline Measurable disease $(+)$ & $92(53 \%)$ & $81(49 \%)$ & \\
\hline No measurable disease (evaluable disease only) & $81(47 \%)$ & $83(51 \%)$ & \\
\hline \multicolumn{4}{|l|}{ Metastatic sites } \\
\hline Peritoneum & $97(56 \%)$ & $89(54 \%)$ & \\
\hline Lymph nodes & $89(51 \%)$ & $95(58 \%)$ & \\
\hline Liver & $45(26 \%)$ & $41(25 \%)$ & \\
\hline Bone & $8(5 \%)$ & $14(9 \%)$ & \\
\hline Lung & $7(4 \%)$ & $8(5 \%)$ & \\
\hline
\end{tabular}

SOX S-1 plus oxaliplatin, SP3 S-1 plus cisplatin (3-weekly), ECOG Eastern Cooperative Oncology Group

${ }^{a}$ Chi-square test

${ }^{\mathrm{b}}$ Student's $t$-test

with $\mathrm{BMI} \geq 25$, SOX demonstrated significantly longer PFS and OS than SP3. In patients aged $\geq 60$ years, the PFS was longer with SOX treatment than SP3; however, the OS was not statistically different between SOX and SP3.

\section{QOL}

The proportion of patients responding to the QoL questionnaire has steadily decreased because of tumor progression or discontinuation of study treatment; the percentage of patients who responded to the questionnaire after the end of the 8th cycle of SOX or SP3 was less than $50 \%$ of the baseline. Therefore, the analysis on the QoL was conducted at the time point of the baseline, after the completion of 2nd, 4th, and 6th cycles of study treatment and at the end of study therapy. During the study treatment, there was no significant difference in all EQ-5D-3L dimensions and VAS between 2 treatment groups (Fig. 3 and Supplementary Table 4).

\section{Safety}

Treatment-related AEs are described in Table 2. Among hematologic AEs (all grades), anemia (30\% versus $22 \%$ ), leucopenia (66\% versus $54 \%$ ), and neutropenia ( $71 \%$ versus $57 \%$ ) developed more commonly with SP3 than with SOX. Thrombocytopenia was more frequent with SOX than with SP3 (71\% versus 58\%). Febrile neutropenia developed in $5 \%$ of SP3-treated patients compared with $0.6 \%$ of SOX-treated patients.

Among non-hematologic AEs (all grades), oral mucositis developed more commonly with SP3 than with SOX. Nausea and vomiting were more common with SOX than with SP3. As expected, peripheral sensory neuropathy was much common with SOX than with SP3 [all grades (59\% versus $35 \%$ ) and grade $\geq 3$ (9\% versus $4 \%$ )]. 
Fig. 2 Survival curves for a progression-free survival and b overall survival (SOX, S-1 plus oxaliplatin; SP3, S-1 plus cisplatin)

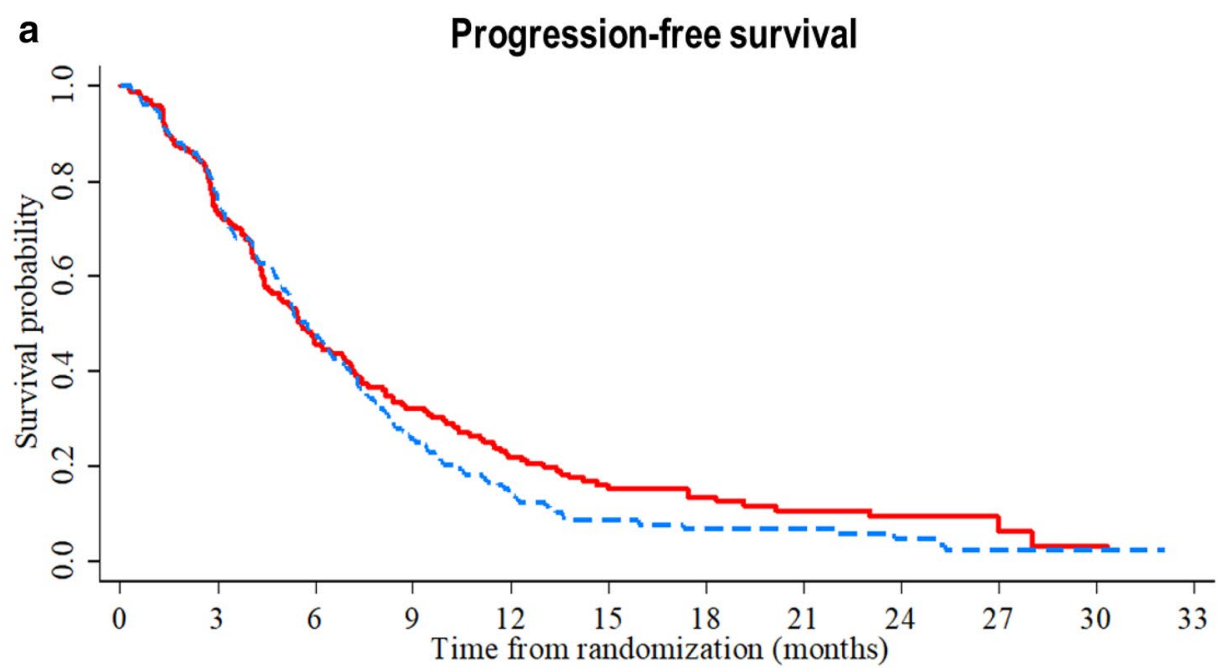

Number at risk

$\begin{array}{lllllllllllll}\text { SOX } & 173 & 120 & 71 & 50 & 33 & 20 & 16 & 10 & 6 & 2 & 1 & 0 \\ \text { SP } & 164 & 114 & 68 & 37 & 20 & 11 & 7 & 7 & 4 & 1 & 1 & 0 \\ & & & & & \end{array}$
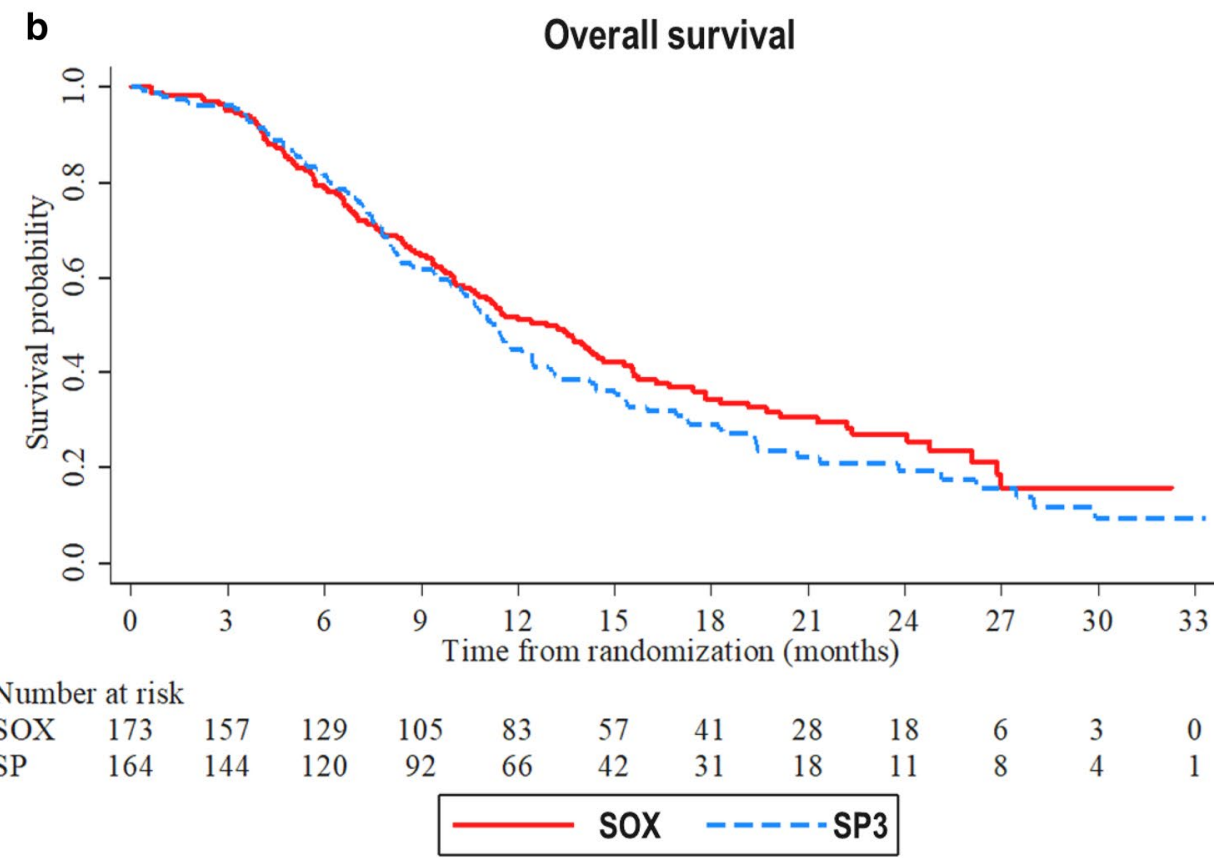

\section{Discussion}

The efficacy, safety, and QOL data of this phase III study (SOPP) were reported. In patients with MRGC, SOX was significantly non-inferior but not superior to SP3 in terms of PFS (median 5.6 versus 5.7 months). Additionally, the OS in both groups did not differ (median 12.9 versus 11.4 months). ORRs were similar between SOX and SP3 (58\% versus $60 \%$ ). The two regimens were well tolerated with different toxicity profiles. The influence of both regimens on QOL showed no significant difference during the treatment period.

For MRGC, palliative chemotherapy improves OS compared with the best supportive care alone. The combination of intravenous 5-fluorouracil and cisplatin ( \pm epirubicin) has been considered the standard first-line chemotherapy in MRGC [2, 5, 6]. Recent trials have shown that oral fluoropyrimidine (capecitabine and S-1) can replace intravenous 5-fluorouracil [5-7] and oxaliplatin can replace cisplatin $[4,5]$. Considering convenience and safety profiles, 
Fig. 3 Changes in quality of life [3-level version of EuroQoL 5-dimensional instrument (EQ5D-3L)] during study treatments: a mobility, b self-care, $\mathbf{c}$ usual activities, $\mathbf{d}$ pain/discomfort, $\mathbf{e}$ anxiety/depression and $\mathbf{f}$ EQ-5D visual analog scale a

\section{Mobility}

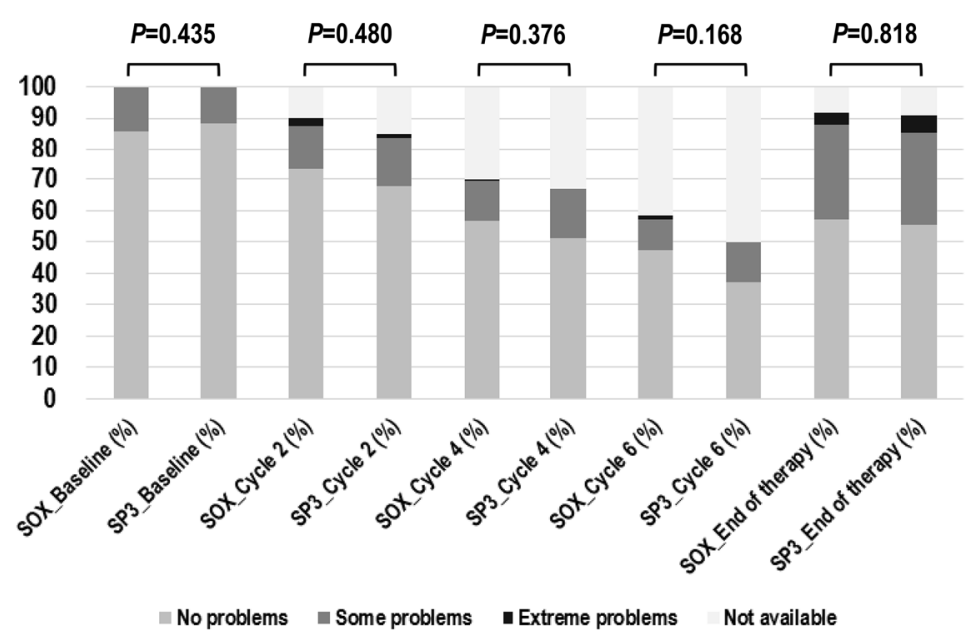

b

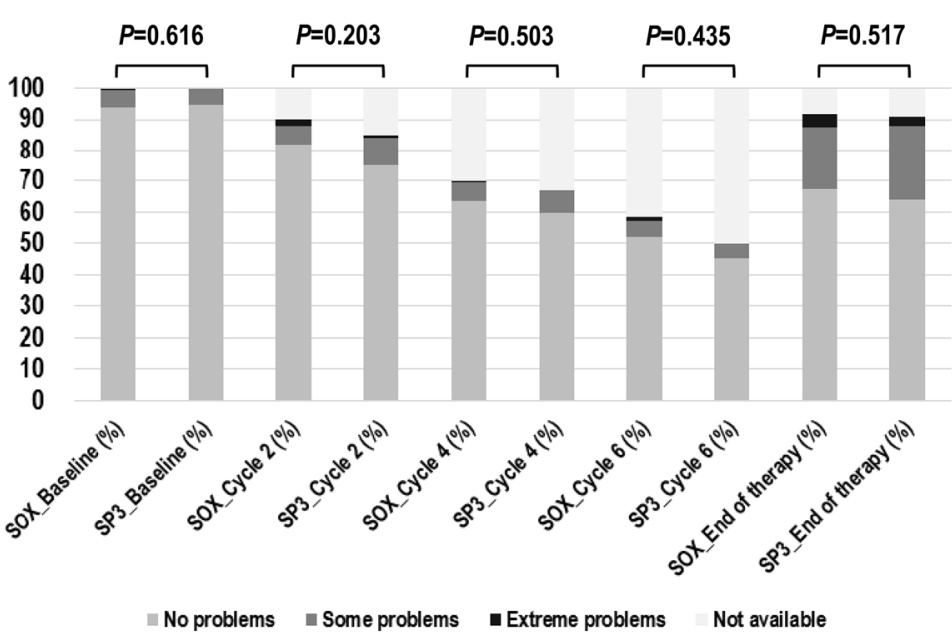

C

\section{Usual Activities}

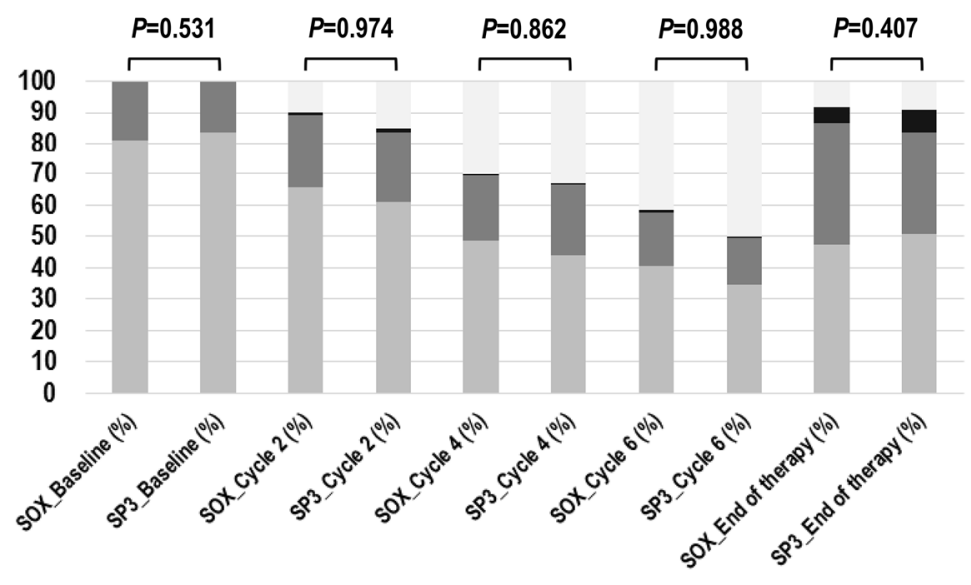


Fig. 3 (continued)

e
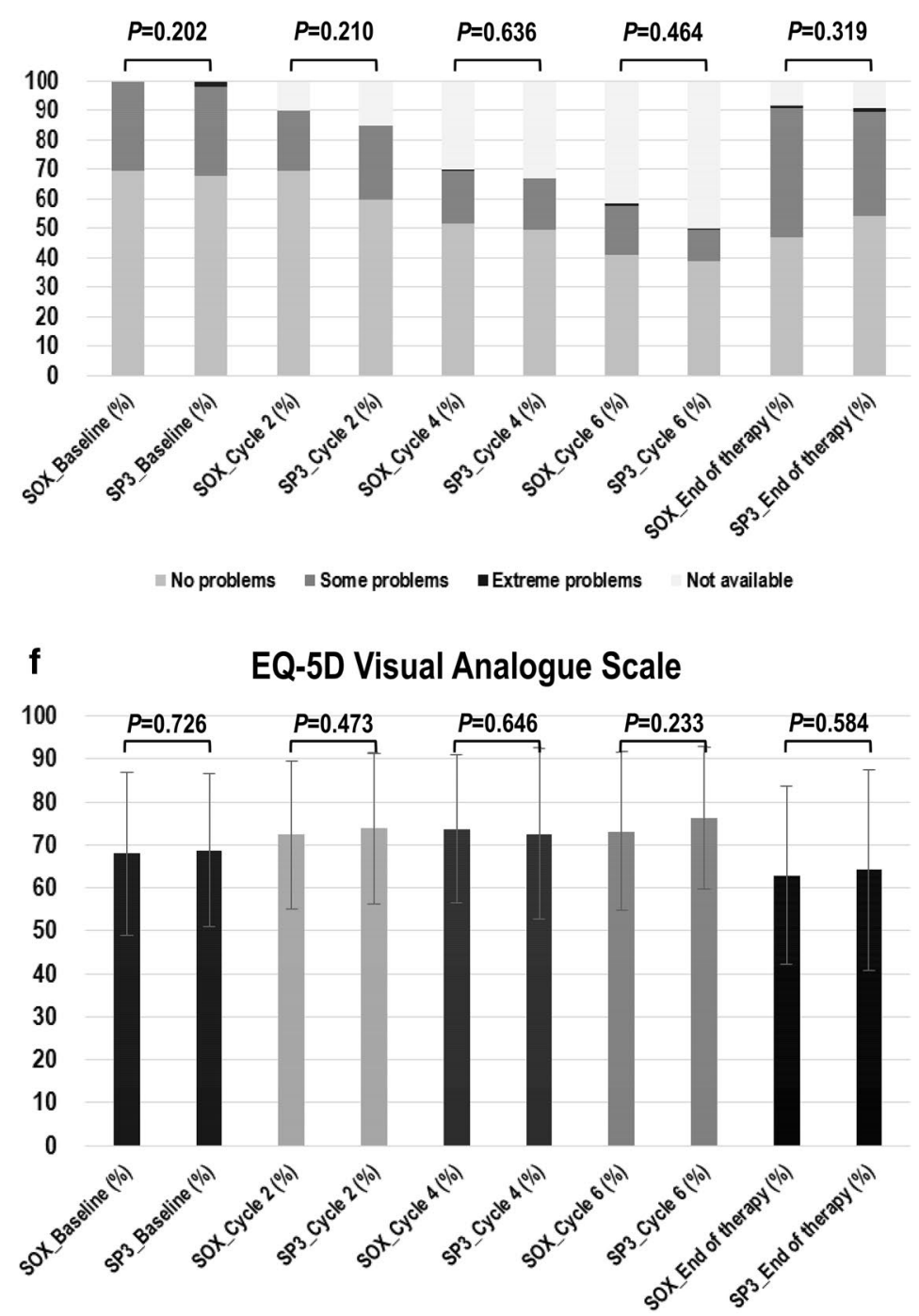
Table 2 Treatment-related adverse events (per patient analysis; $N=337$ )

\begin{tabular}{|c|c|c|c|c|c|c|c|c|c|c|c|c|}
\hline \multirow[b]{2}{*}{ Adverse events } & \multicolumn{6}{|c|}{$\operatorname{SOX}(N=173)$} & \multicolumn{6}{|c|}{ SP3 $(N=164)$} \\
\hline & $\begin{array}{l}\text { Gr. } 1 \\
N\end{array}$ & $\begin{array}{l}\text { Gr. } 2 \\
N\end{array}$ & $\begin{array}{l}\text { Gr. } 3 \\
N\end{array}$ & $\begin{array}{l}\text { Gr. } 4 \\
N\end{array}$ & $\begin{array}{l}\geq \text { Gr. } 3 \\
N(\%)\end{array}$ & $\begin{array}{l}\text { All } \\
N(\%)\end{array}$ & $\begin{array}{l}\text { Gr. } 1 \\
N\end{array}$ & $\begin{array}{l}\text { Gr. } 2 \\
N\end{array}$ & $\begin{array}{l}\text { Gr. } 3 \\
N\end{array}$ & $\begin{array}{l}\text { Gr. } 4 \\
N\end{array}$ & $\begin{array}{l}\geq \text { Gr. } 3 \\
N(\%)\end{array}$ & $\begin{array}{l}\text { All } \\
N(\%)\end{array}$ \\
\hline Anemia & 11 & 18 & 9 & 0 & $9(5.2)$ & $38(22.0)$ & 8 & 23 & 16 & 2 & $18(11.0)$ & $49(29.9)$ \\
\hline White blood cell decreased & 59 & 30 & 3 & 1 & $4(2.3)$ & $93(53.8)$ & 41 & 51 & 13 & 4 & $17(10.4)$ & $109(66.5)$ \\
\hline Neutrophil count decreased & 11 & 60 & 25 & 3 & $28(16.2)$ & $99(57.2)$ & 9 & 43 & 49 & 16 & $65(39.6)$ & $117(71.3)$ \\
\hline Platelet count decreased & 72 & 37 & 10 & 3 & $13(7.5)$ & $122(70.5)$ & 70 & 17 & 5 & 3 & $8(4.9)$ & $95(57.9)$ \\
\hline Febrile neutropenia & 0 & 0 & 1 & 0 & $1(0.6)$ & $1(0.6)$ & 0 & 0 & 6 & 2 & $8(4.9)$ & $8(4.9)$ \\
\hline Fatigue & 51 & 21 & 11 & 0 & $11(6.4)$ & $83(48.0)$ & 53 & 20 & 14 & 0 & $14(8.5)$ & $87(53.0)$ \\
\hline Anorexia & 49 & 24 & 15 & 0 & $15(8.7)$ & $88(50.9)$ & 49 & 32 & 11 & 0 & $11(6.7)$ & $92(56.1)$ \\
\hline Oral mucositis & 23 & 8 & 0 & 0 & $0(0.0)$ & $31(17.9)$ & 30 & 14 & 5 & 0 & $5(3.0)$ & 49 (29.9) \\
\hline Nausea & 68 & 24 & 6 & 0 & $6(3.5)$ & 98 (56.6) & 52 & 15 & 4 & 0 & $4(2.4)$ & $71(43.3)$ \\
\hline Vomiting & 35 & 19 & 2 & 0 & $2(1.2)$ & $56(32.4)$ & 22 & 8 & 3 & 0 & $3(1.8)$ & $33(20.1)$ \\
\hline Diarrhea & 31 & 12 & 7 & 0 & $7(4.0)$ & $50(28.9)$ & 22 & 8 & 6 & 0 & $6(3.7)$ & $36(22.0)$ \\
\hline Constipation & 24 & 14 & 0 & 0 & $0(0.0)$ & $38(22.0)$ & 32 & 11 & 0 & 0 & $0(0.0)$ & $43(26.2)$ \\
\hline Abdominal pain & 16 & 13 & 4 & 0 & $4(2.3)$ & $33(19.1)$ & 12 & 14 & 3 & 0 & $3(1.8)$ & $29(17.7)$ \\
\hline Dyspepsia & 10 & 8 & 0 & 0 & $0(0.0)$ & $18(10.4)$ & 9 & 7 & 0 & 0 & $0(0.0)$ & $16(9.8)$ \\
\hline Dizziness & 12 & 3 & 0 & 0 & $0(0.0)$ & $15(8.7)$ & 14 & 3 & 0 & 0 & $0(0.0)$ & $17(10.4)$ \\
\hline Peripheral sensory neuropathy & 53 & 34 & 15 & 0 & $15(8.7)$ & $102(59.0)$ & 35 & 16 & 5 & 1 & $6(3.7)$ & $57(34.8)$ \\
\hline Skin hyperpigmentation & 12 & 1 & 0 & 0 & $0(0.0)$ & $13(7.5)$ & 21 & 0 & 0 & 0 & $0(0.0)$ & $21(12.8)$ \\
\hline Fever & 8 & 2 & 0 & 0 & $0(0.0)$ & $10(5.8)$ & 13 & 4 & 0 & 0 & $0(0.0)$ & $17(10.4)$ \\
\hline Thromboembolic event & 0 & 1 & 3 & 0 & $3(1.7)$ & $4(2.3)$ & 1 & 2 & 4 & 0 & $4(2.4)$ & $7(4.3)$ \\
\hline Creatinine increased & 1 & 2 & 0 & 0 & $0(0.0)$ & $3(1.7)$ & 2 & 2 & 0 & 0 & $0(0.0)$ & $4(2.4)$ \\
\hline
\end{tabular}

SOX S-1 plus oxaliplatin, SP3 S-1 plus cisplatin (3-weekly), Gr grade

oral fluoropyrimidine and oxaliplatin are being extensively used in MRGC.

S-1 has demonstrated different safety profiles between East Asian and Caucasian patients [17]. According to ethnicity and geographical area, diverse dosing schedules of SP have been used. Based on the result of the SPIRITS trial [10], 5-weekly SP [SP5; S-1 80-120 mg/day (days 1-21) and cisplatin $60 \mathrm{mg} / \mathrm{m}^{2}$ (day 8)] is widely used as first-line chemotherapy for MRGC in Japan. However, 3-weekly SP [SP3; S-1 $80 \mathrm{mg} / \mathrm{m}^{2} /$ day (days $1-14$ ) and cisplatin $60 \mathrm{mg} / \mathrm{m}^{2}$ (day 1)] is popular in Korea [9]. Interestingly, in the Japanese SP5 regimen, the S-1 dose is capped at $120 \mathrm{mg} /$ day if $\mathrm{BSA} \geq 1.5 \mathrm{~m}^{2}$; however, in the Korean SP3 regimen, the S-1 dose $\left(80 \mathrm{mg} / \mathrm{m}^{2} /\right.$ day $)$ is not capped and can be increased to $160 \mathrm{mg} /$ day if $\mathrm{BSA} \geq 2.0$ $\mathrm{m}^{2}$ (Supplementary Table 1). In the Korea-Japan collaborative phase III S-1 Optimal Schedule Study (SOS), SP3 was not only non-inferior but was superior to SP5 in terms of PFS [median 5.5 versus 4.9 months; HR 0.82 (95\% CI, 0.68-0.99)]. However, because the PFS improvement was small and no difference in OS was observed, the Korean and Japanese investigators concluded that both SP3 and SP5 can be recommended as first-line chemotherapy for MRGC [9]. In contrast, considering inter-ethnic variabilities in S-1 tolerability, a 4-weekly regimen was established
[S-1 $50 \mathrm{mg} / \mathrm{m}^{2} /$ day (days $1-21$ ) and cisplatin $75 \mathrm{mg} / \mathrm{m}^{2}$ (day 1)] in Western countries [18].

As S-1 has been popularly used in East Asian patients rather than Western patients, the safety and efficacy of SOX have been actively investigated in East Asian countries. However, the tested dosages and schedules of SOX were diverse in several phase II trials for MRGC [10, 12-15]. In the Japanese G-SOX phase III study $(N=685)$, the oxaliplatin dose was chosen as $100 \mathrm{mg} / \mathrm{m}^{2}$ (day 1) in SOX and the SP5 regimen was used in the reference arm. S-1 was used with the dosage cap at $120 \mathrm{mg} /$ day if $\mathrm{BSA} \geq 1.5 \mathrm{~m}^{2}$ in both SOX and SP5. The investigators demonstrated that SOX is non-inferior to SP5 in terms of PFS [5.5 versus 5.4 months (median); HR 1.004, 95\% CI 0.840-1.199; predefined noninferiority margin 1.30] in the per-protocol population $(N=642)$. The OS [14.1 versus 13.1 months (median)] and ORRs (56\% versus 52\%) did not significantly differ between SOX and SP5 [19]. In this SOPP study, different dosage of oxaliplatin $\left(130 \mathrm{mg} / \mathrm{m}^{2}\right.$; day 1$)$ in the SOX was used and SP3 was chosen as the reference arm. In both SOX and SP3 arms, the $\mathrm{S}-1$ dose $\left(80 \mathrm{mg} / \mathrm{m}^{2} /\right.$ day $)$ was not capped and could be increased to $160 \mathrm{mg} /$ day according to the subject's BSA. This study also demonstrated that SOX is non-inferior to SP3 in terms of PFS [5.6 versus 5.7 months (median); HR 0.85, 95\% CI 0.67-1.07]; when both predefined non-inferiority 
margins of 1.4 and 1.25 were used, SOX was proven to be non-inferior to SP3. OS and ORR did not differ between SOX and SP3 [OS (median), 12.9 versus 11.4 months; ORR, $58 \%$ versus $60 \%$ ]. Therefore, regarding efficacy, although different dosages and treatment schedules of SOX and SP regimens were evaluated, both SOPP and G-SOX studies arrived at the same conclusion.

In terms of severe AEs ( $\geq$ grade 3$)$, anemia ( $11 \%$ versus $5 \%$ ), leucopenia ( $10 \%$ versus $2 \%$ ), neutropenia $(40 \%$ versus $16 \%$ ), and febrile neutropenia ( $5 \%$ versus $0.6 \%$ ) were more common in SP3 than in SOX; conversely, peripheral sensory neuropathy was more common with SOX than SP3 (9\% versus $4 \%$ ). Although thrombocytopenia of all grades was more frequent with SOX than SP3 (71\% versus 58\%), the frequency of severe thrombocytopenia ( $\geq$ grade 3 ) was similar between the 2 arms ( $8 \%$ versus $5 \%$ ). In this study, nausea and vomiting (all grades) were more common with SOX than SP3. However, in the G-SOX study, nausea (all grades) was more frequent with SP5 than SOX. This differential pattern in the incidence of nausea and vomiting could be attributed to the different oxaliplatin doses used in the two studies. Although some differences exist in the pattern of developing treatment-related AEs between the SOPP and G-SOX studies, the overall differences in the AE profiles observed with SOX and SP in this SOPP study were consistent with those observed in the G-SOX study [19].

Interestingly, PFS and OS curves of SOX and SP3 tended to separate at about 7 and 10 months after randomization, respectively. This trend was not observed in the G-SOX study. At the end of this study, the study treatment was still ongoing in 16 subjects (9\%) in SOX and 5 (3\%) in SP3 (Fig. 1). Between the two regimens, there was no difference in the delivery of platinum agents. However, although there was no statistical difference, S-1 in SOX was delivered longer than S-1 in SP3 [median treatment cycles of S-1, 6 (range 1-45) in SOX versus 5 (range 1-35) in SP3; mean treatment cycles of S-1, 8.6 in SOX versus 7.2 in SP3 (Supplementary Table 2)]. When looked into the causes of study treatment discontinuation (Fig. 1), the proportion of subjects who were dropped out of study treatment owing to adverse events or refusal of treatment due to toxicity was numerically higher in SOX (9\%) than in SP3 (5\%). However, unexpectedly, subjects in SP3 rejected the study treatment more frequently for reasons other than identified toxicities (9\%) than those in SOX (3\%). Although the exact reasons of more treatment refusal in SP3 than in SOX was not clear, it is thought that there is still a possibility that treatment compliance decreased more in SP3 than in SOX due to unrecognized adverse events or other reasons. In addition, it was found that 13 subjects received conversion gastrectomy ( 9 in SOX and 4 in SP3) after study treatment (Supplementary Table 5); all these subjects had favorable OS outcomes and the median OS of these subjects did not reach. It is thought that the role of conversion surgery after palliative chemotherapy is not yet established in MRGC, so more researches are necessary. Although these explanations may not be enough, more conversion gastrectomy and more prolonged administration of S-1 in SOX group than in SP3 group may have contributed to the separation of PFS and OS curves after the observed time points between SOX and SP3.

In this study, subgroup analyses indicated no overall differences in PFS and OS between SOX and SP3. However, patients with a BMI $\geq 25$ or age $\geq 60$ years demonstrated longer PFS or OS with SOX than with SP3 (Supplementary Fig. 1). In the G-SOX study, the investigators have reported that patients with peritoneal metastasis had significantly longer OS with SOX than with SP5; the investigators could not observe any other subgroups with significantly improved OS with either SOX or SP5. The G-SOX investigators did not include BMI as one of the parameters for subgroup analyses of survival. In contrast, organs (such as the liver, lungs, or peritoneum) involved by metastasis were not included as parameters for subgroup analyses of PFS or OS in our study. Therefore, a direct comparison of subgroup analyses is not feasible between G-SOX and the current studies.

This study has some limitations. First, the study treatments were not blinded and $49 \%$ of enrolled subjects (164/337) had no measurable target lesion. The fact that only investigators, without the confirmation of independent central reviewers, determined tumor progression might be a limitation. Second, some experts may criticize the noninferiority margin 1.4 as the basis for sample size calculation in this study was too wide. However, since non-inferiority margins of both 1.4 and 1.25 were satisfied through the twostep tests, it is thought that SOX was sufficiently proven non-inferior in terms of PFS compared to SP3. Third, some experts may question whether it is appropriate to set the primary endpoint to PFS in a phase III clinical trial of first-line chemotherapy in MRGC. In a meta-analysis of the GASTRIC group, the investigators showed that chemotherapy effects on PFS and OS were only moderately correlated, and thus they could not confirm the validity of PFS as a surrogate endpoint for OS in MRGC [20]. It is agreed that PFS is not a good surrogate endpoint for OS in superiority trials for MRGC. However, as in other tumors, OS is greatly influenced by salvage chemotherapy after first-line chemotherapy, and the salvage chemotherapy is influenced by regional difference and practice pattern of the attending physicians [21]. In addition, as the efficacy of salvage chemotherapy has been recently demonstrated in MRGC, the frequency of using second-line or later-line chemotherapy is increasing [22-24]. Therefore, it is believed that PFS as a primary endpoint is practically acceptable in non-inferiority trials of first-line chemotherapy in MRGC. For example, PFS was previously used as a primary endpoint in a phase III trial (ML17032), which had shown the non-inferiority of 
capecitabine/cisplatin compared to 5-fluorouracil/cisplatin in MRGC [6].

In conclusion, this SOPP study demonstrated that SOX is non-inferior to SP3. The two regimens were well tolerated with different toxicity profiles. Considering the SOPP and Japanese G-SOX studies, the SOX regimen can be recommended as a first-line treatment of MRGC in East Asian countries.

Acknowledgements The authors thank all patients and investigators who participated in this study. They also thank Jeil Pharmaceutical Co., Ltd (Korea) for the supply of oxaliplatin.

Funding This study was partly supported by Jeil Pharmaceutical Co., Ltd. This was an investigator-initiated trial. The company had no role in the interpretation of data or the preparation, review, or approval of the manuscript, or decision to submit the study for publication. The corresponding author had full access to all study data and was responsible for the final decision to submit for publication.

\section{Compliance with ethical standards}

Conflict of interest K-WL reported honorarium from Bristol Myers Squibb, Eli Lilly, and Genexine, outside the submitted work; advisory role with Bayer outside the submitted work; and research funding from AstraZeneca/MedImmune, ASLAN pharmaceuticals, Array BioPhrama, ALX Oncology, BeiGene, Daiichi Sankyo, Five Prime Therapeutics, Green Cross, LSK BioPharma, MacroGenics, Merck KGaA, Merck Sharp \& Dohme, Ono Pharmaceutical, Pharmacyclics, Pfizer, Taiho Pharmaceutical, and Zymeworks to his institution (for conducting clinical trials), outside the submitted work. M-HR reported honorarium or advisory role with ONO Pharmaceutical, Bristol Myers Squibb, Merck Sharp \& Dohme, Eli Lilly, Taiho, Novartis, and Daehwa Pharmaceutical, outside the submitted work. Y-KK reported advisory role with Ono Pharmaceutical, Bristol Myers Squibb, Amgen, Daehwa Pharmaceutical, ALX Oncology, Zymeworks, Novartis, Macrogenics, and Surface Oncology, outside the submitted work. All the remaining authors (I-JC, YIP, B-HN, H-SO, KHL, HSH, B-GS, J-CJ, HRL, JWK, SRP, and SHC) have declared no conflicts of interest.

\section{References}

1. Ferlay J, Soerjomataram I, Dikshit R, Eser S, Mathers C, Rebelo $\mathrm{M}$, et al. Cancer incidence and mortality worldwide: sources, methods and major patterns in GLOBOCAN 2012. Int J Cancer. 2015;136:E359-386.

2. Kim JW, Kim JG, Kang BW, Chung IJ, Hong YS, Kim TY, et al. Treatment patterns and changes in quality of life during first-line palliative chemotherapy in korean patients with advanced gastric cancer. Cancer Res Treat. 2019;51:223-39.

3. Bang YJ, Van Cutsem E, Feyereislova A, Chung HC, Shen L, Sawaki A, et al. Trastuzumab in combination with chemotherapy versus chemotherapy alone for treatment of HER2-positive advanced gastric or gastro-oesophageal junction cancer (ToGA): a phase 3, open-label, randomised controlled trial. Lancet. 2010;376:687-97.

4. Al-Batran SE, Hartmann JT, Probst S, Schmalenberg H, Hollerbach S, Hofheinz R, et al. Phase III trial in metastatic gastroesophageal adenocarcinoma with fluorouracil, leucovorin plus either oxaliplatin or cisplatin: a study of the Arbeitsgemeinschaft Internistische Onkologie. J Clin Oncol. 2008;26:1435-42.
5. Cunningham D, Starling N, Rao S, Iveson T, Nicolson M, Coxon $\mathrm{F}$, et al. Capecitabine and oxaliplatin for advanced esophagogastric cancer. N Engl J Med. 2008;358:36-46.

6. Kang YK, Kang WK, Shin DB, Chen J, Xiong J, Wang J, et al. Capecitabine/cisplatin versus 5-fluorouracil/cisplatin as first-line therapy in patients with advanced gastric cancer: a randomised phase III noninferiority trial. Ann Oncol. 2009;20:666-73.

7. Boku N, Yamamoto S, Fukuda H, Shirao K, Doi T, Sawaki A, et al. Fluorouracil versus combination of irinotecan plus cisplatin versus $\mathrm{S}-1$ in metastatic gastric cancer: a randomised phase 3 study. Lancet Oncol. 2009;10:1063-9.

8. Koizumi W, Narahara H, Hara T, Takagane A, Akiya T, Takagi M, et al. S-1 plus cisplatin versus $S-1$ alone for first-line treatment of advanced gastric cancer (SPIRITS trial): a phase III trial. Lancet Oncol. 2008;9:215-21.

9. Ryu MH, Baba E, Lee KH, Park YI, Boku N, Hyodo I, et al. Comparison of two different $\mathrm{S}-1$ plus cisplatin dosing schedules as first-line chemotherapy for metastatic and/or recurrent gastric cancer: a multicenter, randomized phase III trial (SOS). Ann Oncol. 2015;26:2097-101.

10. Koizumi W, Takiuchi H, Yamada Y, Boku N, Fuse N, Muro K, et al. Phase II study of oxaliplatin plus S-1 as first-line treatment for advanced gastric cancer (G-SOX study). Ann Oncol. 2010;21:1001-5.

11. Park I, Lee JL, Ryu MH, Chang HM, Kim TW, Sym SJ, et al. Phase I/II and pharmacokinetic study of S-1 and oxaliplatin in previously untreated advanced gastric cancer. Cancer Chemother Pharmacol. 2010;65:473-80.

12. Kim GM, Jeung HC, Rha SY, Kim HS, Jung I, Nam BH, et al. A randomized phase II trial of S-1-oxaliplatin versus capecitabine-oxaliplatin in advanced gastric cancer. Eur J Cancer. 2012;48:518-26.

13. Oh SY, Kwon HC, Jeong SH, Joo YT, Lee YJ, Cho S, et al. A phase II study of S-1 and oxaliplatin (SOx) combination chemotherapy as a first-line therapy for patients with advanced gastric cancer. Invest New Drugs. 2012;30:350-6.

14. Yang L, Song Y, Zhou AP, Qin Q, Chi Y, Huang J, et al. A phase II trial of oxaliplatin plus S-1 as a first-line chemotherapy for patients with advanced gastric cancer. Chin Med J (Engl). 2013;126:3470-4.

15. Xiao C, Qian J, Zheng Y, Song F, Wang Q, Jiang H, et al. A phase II study of biweekly oxaliplatin plus S-1 combination chemotherapy as a first-line treatment for patients with metastatic or advanced gastric cancer in China. Medicine (Baltimore). 2019;98:e15696.

16. Rabin R, de Charro F. EQ-5D: a measure of health status from the EuroQol Group. Ann Med. 2001;33:337-43.

17. Chuah B, Goh BC, Lee SC, Soong R, Lau F, Mulay M, et al. Comparison of the pharmacokinetics and pharmacodynamics of S-1 between Caucasian and East Asian patients. Cancer Sci. 2011;102:478-83.

18. Ajani JA, Rodriguez W, Bodoky G, Moiseyenko V, Lichinitser $\mathrm{M}$, Gorbunova V, et al. Multicenter phase III comparison of cisplatin/S-1 with cisplatin/infusional fluorouracil in advanced gastric or gastroesophageal adenocarcinoma study: the FLAGS trial. J Clin Oncol. 2010;28:1547-53.

19. Yamada Y, Higuchi K, Nishikawa K, Gotoh M, Fuse N, Sugimoto N, et al. Phase III study comparing oxaliplatin plus S-1 with cisplatin plus $\mathrm{S}-1$ in chemotherapy-naive patients with advanced gastric cancer. Ann Oncol. 2015;26:141-8.

20. Paoletti X, Oba K, Bang YJ, Bleiberg H, Boku N, Bouche O, et al. Progression-free survival as a surrogate for overall survival in advanced/recurrent gastric cancer trials: a meta-analysis. J Natl Cancer Inst. 2013;105:1667-700.

21. Sawaki A, Yamada Y, Yamaguchi K, Nishina T, Doi T, Satoh T, et al. Regional differences in advanced gastric cancer: exploratory 
analyses of the AVAGAST placebo arm. Gastric Cancer. 2018;21:429-38.

22. Kim HS, Kim HJ, Kim SY, Kim TY, Lee KW, Baek SK, et al. Second-line chemotherapy versus supportive cancer treatment in advanced gastric cancer: a meta-analysis. Ann Oncol. 2013;24:2850-4.

23. Kang YK, Boku N, Satoh T, Ryu MH, Chao Y, Kato K, et al. Nivolumab in patients with advanced gastric or gastro-oesophageal junction cancer refractory to, or intolerant of, at least two previous chemotherapy regimens (ONO-4538-12, ATTRAC TION-2): a randomised, double-blind, placebo-controlled, phase 3 trial. Lancet. 2017;390:2461-71.
24. Shitara K, Doi T, Dvorkin M, Mansoor W, Arkenau HT, Prokharau A, et al. Trifluridine/tipiracil versus placebo in patients with heavily pretreated metastatic gastric cancer (TAGS): a randomised, double-blind, placebo-controlled, phase 3 trial. Lancet Oncol. 2018;19:1437-48.

Publisher's Note Springer Nature remains neutral with regard to jurisdictional claims in published maps and institutional affiliations.

\section{Affiliations}

Keun-Wook Lee ${ }^{1} \cdot$ Ik-Joo Chung $^{2} \cdot$ Min-Hee Ryu ${ }^{3} \cdot$ Young lee Park ${ }^{4} \cdot$ Byung-Ho Nam $^{5,12} \cdot \mathrm{Ho}^{-S u k} \mathrm{Oh}^{6} \cdot$

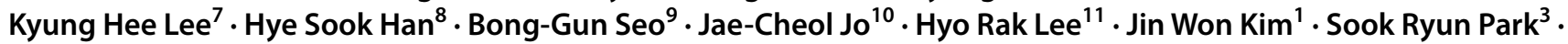
Sang Hee $\mathrm{Cho}^{2}$. Yoon-Koo $\mathrm{Kang}^{3}$ on behalf of the SOPP study investigators

1 Department of Internal Medicine, Seoul National University Bundang Hospital, Seoul National University College of Medicine, Seongnam, Republic of Korea

2 Department of Hematology-Oncology, Chonnam National University Hwasun Hospital, Chonnam National University Medical School, Hwasun, Republic of Korea

3 Department of Oncology, Asan Medical Center, University of Ulsan College of Medicine, Seoul, Republic of Korea

4 Center for Gastric Cancer, Research Institute and Hospital, National Cancer Center, Goyang, Republic of Korea

5 Department of Cancer Control and Policy, Graduate School of Cancer Science and Policy, National Cancer Center, Goyang, Republic of Korea

6 Department of Internal Medicine, Gangneung Asan Hospital, University of Ulsan College of Medicine, Gangneung, Republic of Korea
7 Department of Medicine, Yeungnam University College of Medicine, Daegu, Republic of Korea

8 Department of Internal Medicine, College of Medicine, Chungbuk National University, Cheongju, Republic of Korea

9 Department of Internal Medicine, Dongnam Institute of Radiological and Medical Sciences, Busan, Republic of Korea

10 Department of Internal Medicine, Ulsan University Hospital, University of Ulsan College of Medicine, Ulsan, Republic of Korea

11 Department of Internal Medicine, Korea Cancer Center Hospital, Seoul, Republic of Korea

12 HERINGS, Seoul, Republic of Korea 